\title{
Definition of product requirements of a MR compatible bone biopsy system using workflow analysis
}

\author{
A. Boese ${ }^{1}$, M. Schmidt ${ }^{1}$, T. Neumuth ${ }^{2}$, G. Rose $^{1}$ \\ ${ }^{1}$ Otto-von-Guericke University, Department of Healthcare Telematics and Medical Technology, Magdeburg, Germany, \\ axel.boese@ovgu.de \\ ${ }^{2}$ University Leipzig, Innovation Center Computer Assisted Surgery (ICCAS), Leipzig, Germany
}

\section{Introduction}

Image-guided needle biopsy is a standard method in cancer diagnostic for tumor staging [1, 2]. As guiding modality computer tomography (CT), ultrasound, X-ray or magnetic resonant imaging (MRI) is being used for instrument navigation. For MRI guided procedures a MRI compatible needle in combination with a driller is required. To define the specification of such a system workflow analysis was applied as part of the product development cycle [3].

\section{Methods}

Three bone biopsies under CT guidance as the standard imaging modality and one mama biopsy under MRI guidance were tracked and recorded. For the workflow analysis a simple paper and pen combination as well as the SWAN workflow editor [4] with advanced functionality were used. The procedure itself, the interaction with the imaging system and the visualization of the instrument were documented. This was determined as the main challenge, because fully MRI compatible materials like ceramics are poorly identifiable [5] within the images. Since the properties of these materials are different from the traditionally used steel, the workflow itself is to change to fix problems like loss of the drilled bore in the bone or pain during insertion of the biopsy set.

\section{Results}

Based on the captured information a general concept for a bone biopsy system for MRI guided interventions was set up. The general requirements like dimensions of biopsy set, steps of intervention or handling aspects were defined. The workflow itself was adapted to the use of MR imaging, since thereby manipulation of the instruments during imaging is possible.

\section{Conclusion}

Workflow analysis is a well accepted method to get a general understanding of medical procedures. The main functionalities, deficits of used equipment as well as unobvious side aspects can be detected and kept into a product development process. In addition to this the state of the art of medical interventions can be documented.

\section{References}

[1] J. H. Linebarger, J. Landercasper, R. L. Ellis, J. D. Gundrum, K. A. Marcou, B. M. De Maiffe, J. M. Hudak, and J. J. Andersen, "Core needle biopsy rate for new cancer diagnosis in an interdisciplinary breast center: evaluation of quality of care 2007-2008," Ann. Surg., vol. 255, no. 1, pp. 38-43, Jan. 2012.

[2] S. C. Adams, B. K. Potter, D. J. Pitcher, and H. T. Temple, "Office-based core needle biopsy of bone and soft tissue malignancies: an accurate alternative to open biopsy with infrequent complications," Clin. Orthop. Relat. Res., vol. 468, no. 10, pp. 2774-2780, Oct. 2010.

[3] A. Boese and K.-H. Grote, "Workflow Analysis as Tool for Development of Medical Devices, a white Paper," in Proceedings of The World Congress on Engineering 2010, Vol III, pp2149-2152, London, U.K., 2010.

[4] T. Neumuth, G. Strauß, J. Meixensberger, H. U. Lemke, O. Burgert, “Acquisition of Process Description from Surgical Interventions," 2006.

[5] H. Matsuura, T. Inoue, K. Ogasawara, M. Sasaki, H. Konno, Y. Kuzu, H. Nishimoto, and A. Ogawa, “Quantitative analysis of magnetic resonance imaging susceptibility artifacts caused by neurosurgical biomaterials: comparison of 0.5, 1.5, and 3.0 Tesla magnetic fields," Neurol. Med. Chir. (Tokyo), vol. 45, no. 8, pp. 395-398; discussion 398-399, Aug. 2005. 